\title{
EventNet: Inferring Temporal Relations Between Commonsense Events
}

\author{
Jose Espinosa $^{1}$, Henry Lieberman ${ }^{1}$ \\ ${ }^{1}$ MIT Media Lab, Software Agents Group, 20 Ames St., \\ Cambridge, Massachusetts, 02139, USA \\ \{jhe, lieber\}@media.mit.edu \\ http://agents.media.mit.edu
}

\begin{abstract}
In this paper, we describe EventNet, a toolkit for inferring temporal relations between Commonsense events. It comprises 10,000 nodes and 30,000 temporal links mined from the Openmind Commonsense Knowledge Base. It enables applications to deduce "obvious" (to people) temporal relations between commonly occurring events, for example: First, you wake up, then you can leave the house in the morning. The temporal relation might be one of cause and effect, of action/goal or prerequisite relations, or simply that they tend to follow each other in a commonly occurring "script". In addition, the algorithm has some built-in heuristics to infer when its information is not enough. It then finds semantically similar nodes to dynamically search the knowledge base. EventNet has been used in projects such as an intelligent kitchen, and in intelligent interfaces for consumer electronics devices.
\end{abstract}

\section{Introduction}

Building applications that successfully interact with humans in everyday life requires considerable knowledge about the world, people, and everyday life. These applications need to know about people's goals, motivations and desires. We'd like to infer that if a person is filling a carafe with water just after waking up, it's likely that the person is making coffee; and the effect of drinking coffee is to become more awake. This allows building systems that provide more proactive interaction with users.

This paper focuses on one particular aspect of Commonsense knowledge - understanding temporal relations between events. Temporal reasoning allows an interface to predict what might happen next, enabling proactive interfaces that automatically make suggestions that the user might find pertinent to upcoming situations. It allows a system to infer antecedents - infer from the present state what has likely led up to that state. Antecedent events allow the system to fill in gaps in incomplete explicit information supplied by the 
user. It also permits the system to understand events that might take place concurrently with the current event.

We extract temporal information from a more general knowledge base concerning Commonsense knowledge in general, Open Mind Common Sense [1], described in the next section. We then turn specifically to EventNet, where we show how we extract temporal event information and provide a simple spreading-activation inference algorithm to propagate temporal relations. We then describe application-oriented tools, including a plan recognizer. Finally, we describe some specific application projects, such as improved interfaces to networks of consumer electronics devices, and an intelligent kitchen.

\subsection{Openmind Commonsense Project}

Giving computers commonsense has been one of the biggest goals of Artificial Intelligence since the beginning. A program should know that in order to use your car it should be in the same place you are (or at least at reasonable distance) [2]. To accomplish this goal it is necessary to solve two main problems: a) collecting and storing all the knowledge, and b) building reasoning algorithms. The largest and longest-standing effort in this direction is Lenat's CYC [3]. CYC is produced by a team of knowledge engineers who have worked for two decades to carefully encode Common Sense in a formal language, CYCL.

In contrast, our Openmind Commonsense Project collects common sense knowledge from volunteers over the web. It is a website where the users are asked to fill templates like "Something that might happened when you go to is that you might using plain English [1]. By the spring of 2005, the site has collected around 750,000 sentences from 16,000 contributors. An example of the knowledge find in this project is "Something that might happen when you go to the zoo is that you might see exotic animals" and "The effect of walking in the rain is getting wet."

By mining the templates in OpenMind and parsing techniques, a semantic network, called ConceptNet, was created [4]. This network has 300,000 nodes and 1.6 millions links, like [Subevent "go to the zoo" "see exotic animals"] or [EffectOf "walk in the rain" "get wet"]. ConceptNet has operations like getting the context of a given topic, analogy making, topic spotting, and classification [4]. ConceptNet has been used to embed commonsense reasoning into interactive applications [5].

Using the temporal links from ConceptNet a dynamic Bayesian network called LifeNet is created by "egocentric" nodes of the form "I go to the zoo" and a set of probabilities linking the nodes. This network uses belief propagation [6] to perform a variety of temporal operations like predicting what else might be true now, in the near future or in the near past, explaining why some event happened, or filtering nodes that are not likely to be true. Due to its semantic imprecision, we introduce probabilistic reasoning into LifeNet $[7]$. 


\section{EventNet}

EventNet uses the temporal nodes in LifeNet to create an association network. It can make predictions of the more likely preceding or subsequent events associated with a certain set of events, in contrast to LifeNet's single-event predictions. Also, it can compute paths between two events. It is able to infer that, in order to watch a movie, it is necessary to buy a ticket, and that a person is likely to buy popcorn. EventNet is a suitable inference engine for applications that have to watch users' actions and give them advice or suggestions. (See section 3 for current examples).

The EventNet links are expressed as triplets of the form (0.504 "I go to a zoo" "I see exotic animals"). The first element is the weight of the link, the second the parent (preceding) node and the third, the son (subsequent) node. In this network all the nodes are expressed in an egocentric way with no distinctions between the subject executing or receiving the action. Examples of EventNet nodes are "I run," "I eat breakfast," or "I am sick."

\subsection{EventNet inference algorithm}

EventNet uses a spreading activation algorithm to do its inferences. At each step, every energized node spreads a fraction of its energy to its adjacent nodes. The value of the spread energy is directly proportional to the weight between the nodes.

The energy of any node after a spreading step is calculated using the formula,

$$
n_{i}=\bigsqcup_{j=\operatorname{links}\left(n_{i}\right)} \operatorname{energy}\left(n_{j}\right) * \text { weight }\left(n_{i}, n_{j}\right)
$$

This causes the nodes with high connectivity to increase their likelihood, filtering out the irrelevant or noise links. Spreading energy from the node "I rain" will reach the concepts "I paint someone's house," "I get wet," "I go to baseball game," "I walk in rain," "I go to zoo" in the first iteration. In the second iteration the top-ten concepts are "I walk in rain," "I catch a cold," "I get wet," "I am cold," "I wash someone's car," "I wash away dirt," "I have clothes to clean," "I repair my umbrella," "I play in water," "I have a bath." Note that the unlikely nodes ("I paint someone's house" and "I go to a baseball game") are filtered out in the second iteration (Fig. 1).

Despite EventNet's origin in a Bayesian network, it uses spreading activation instead of a Bayes-rule inference algorithm. The latter method does not allow changing the topology of the network during the inference; this is needed to add the semantic links. Also, spreading activation emphasizes highly connected nodes by adding multiple paths while Bayes networks subtracts the extra overlap. 


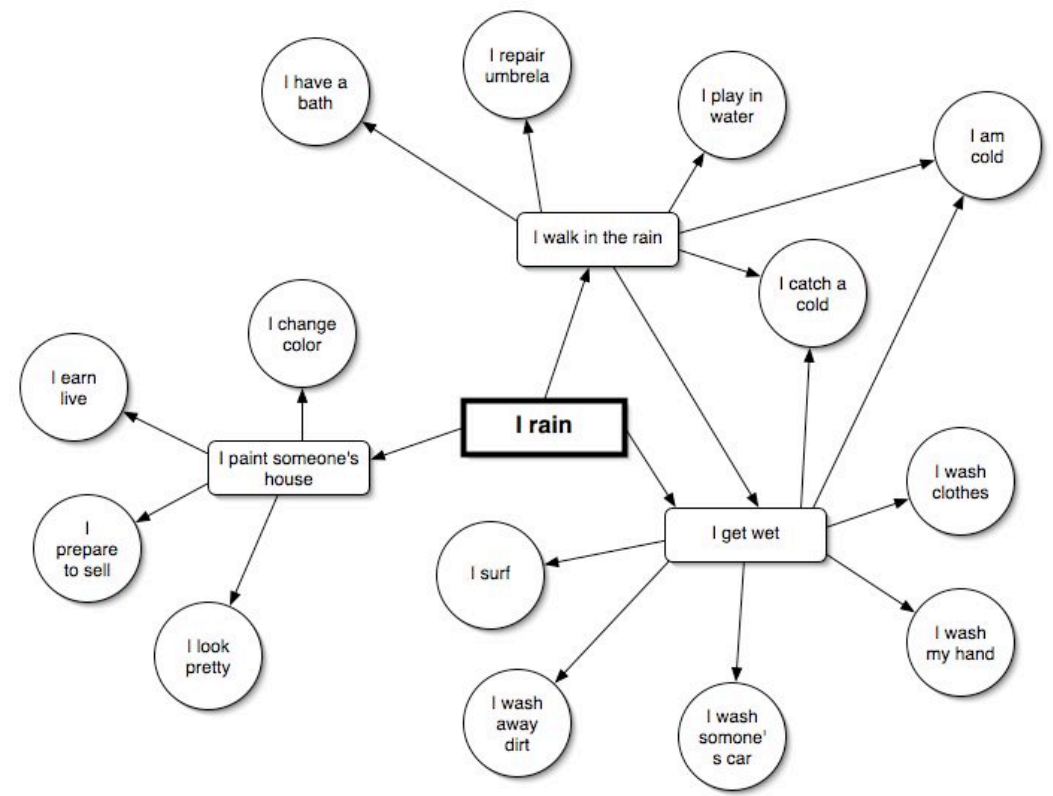

Fig. 1. Spreading activation over one node. The bold square node is the query node, the rounded-rectangle nodes are the nodes reached on the first iteration. The circular nodes are reached in the second iteration. The unlikely node "I paint someone's house" is de-empasized, in contrast to the more plausible "I get wet" and "I walk in the rain"

\subsection{EventNet temporal toolkit}

EventNet is designed to be a temporal reasoning toolkit. It provides two basic operations: plan recognition and paths between events. EventNet is implemented in Common Lisp; in addition the main API has been packaged as an XML-RPC server to be easily accessed by external applications. It provide two basic operations: 
Plan recognizer. This operation is able to infer what are the user's next actions or goals based on a set of observed events. This operation works by applying a certain amount of energy $\square_{\text {node }}$ to one or more observed events, then applying the spreading activation algorithm described in the previous section. This operation infers a set of possible next events, previous events or temporal related events. The future events are calculated by spreading the energy forward from the parents to its sons. The past events are calculated by spreading the energy backward from the sons to its parents. The temporally related events treat EventNet as a undirected graph, giving you the composition of the next and past events.

The API for the plan recognizer allows specifying the times the spreading step will be applied before giving the answer and the desired size of the answer. For example: calling the function (find-next-state "wake up" :size 100 :ntimes 2) gives you the top-100 ranked nodes associated with future events of "wake up" after speeding the node two times. EventNet returns the top 100 temporally related nodes in 0.001 seconds average using a PowerPC G4 500 MHz, 1024MB Ram, Mac OS X 10.2.8 and Macintosh Common Lisp 5.0.

Paths between events: This operation finds a plausible explanation of a temporal relation, as a sequence of temporally related events between the two. This algorithm is inspired on a planning algorithm originally proposed by Maes [8].

The algorithm works as follows:

1. Each of the source and goal nodes is excited with energy $\square_{\text {source }}$ and $\square_{\text {goal }}$ respectively. In addition, the source nodes are marked as activated.

2. The source nodes spread energy to their children and the goal nodes spread energy to their parents. The amount of energy injected is directly proportional to the current energy of the node and the weight of the link, as expressed in function 1.

3. All the nodes that have received energy from at least one of their parents keep spreading the energy to their sons. In the same way, all the nodes that have received energy from at least one of their sons keep spreading the energy to their parents.

4. After each spreading step, the energy of the nodes is averaged to a certain value $\square$. This step keeps the total energy within the network constant.

5. All the nodes that have at least one of their parents marked as activated and their energy above a threshold $\square$ are marked as active. If no nodes are marked as activated the threshold $\square$ is decreased by $10 \%$.

6. If in a single iteration no new nodes are excited, there are no paths between the source and goal nodes. In this case the network uses the semantic information within the nodes to generate a new link and connect the two otherwise unconnected nodes. See the explanation about the semantic link calculus below. 
7. Repeat steps $2,3,4,5$, and 6 until there is at least one path of activated nodes between the source node and the goal node.

This operation provides an explanation of plausible events, without committing to whether the events are necessary or just stereotypical. A path between the event "I wake up" and "I go to work" can be "I wake up," "I take a bath," "I put my clothes on," "I eat breakfast," "I drive my car," and "I go to work." In this path just the node "I put my clothes on" is necessary to accomplish the goal, but it is safe to assume that the other nodes are also true.

Create semantic links. EventNet is formed by 10,000 nodes and 30,000 links. It is not a fully connected graph; there is not always a path between two nodes. To bridge this gap, the system can dynamically create new links between two nodes that are semantically similar. This similarity is calculated using synonyms from WordNet [9] and analogies from ConceptNet [4]. This operation is an extension of Cohen's WHIRL [10] using WordNet's and ConceptNet's semantic information.

For example, we try to find a path between the nodes "I get money" and "I eat lunch", but the nodes are not directly connected. The algorithm generates new links based on the semantic similarity of the nodes. In this case the nodes "I buy pizza" is semantically linked with the nodes "I have hamburger" and "I have hot dog". This semantic expansion also is used to match existing nodes to process text queries, getting a close match by semantic similarity rather than plain keyword matching.

\section{Using EventNet in interactive applications}

The plan recognizer function of EventNet is used to infer a set of actions, goals and events based on the user's actions. Some current applications include: reducing complexity of consumer electronic interfaces, a kitchen scheduler, and an integrated calendar/to-do list.

\subsection{Reducing complexity of consumer electronics interfaces}

Current consumer electronic interfaces are difficult to use because the designers try to map an increasing number of functions of the device to a limited set of buttons. So, when the designers try to map to many functions to a handful of buttons they have to create menus, modes, push-and-hold buttons; these are the kind of interactions that frustrate the user.

We are exploring a new approach to interaction, where the devices are continually watching the users actions and proactively proposing actions that might satisfy the user's goals. We use EventNet's plan recognizer as an inference engine for this application. Furthermore, the devices are able to propose actions necessary to reach the users goals, execute them automatically, or self-debug when things go wrong. 
For example: when the user plugs a guitar into the amplifier, the system asks EventNet for the next actions after the event "I plug my guitar". The answer to the query contains the nodes "I play music" and "I record a song," among others. These nodes trigger the device's functions for playing and recording music. Since EventNet doesn't make a distinction between goals and events, the device's functions are considered actions. This heuristic will filter out the nodes that cannot de executed by the system. For example, the node "I dance" is retrieved but it is ignored since there is no device function capable of dancing.

In addition, the system can suggest device functions according to the user's expressed goals; if the user says, "I want to watch the news," the system is able to recognize this as a goal and ask EventNet for the events preceding by the node "I watch the news." The answer that comes out from EventNet makes the system suggest the device actions watchtelevision and hear-radio. Later, the user can complain "Why I am not hearing anything?" The system uses the path-finding algorithm of EventNet between the current goal ("I hear the radio") and the user's desired action "I hear." The EventNet answer is compared to the state of the system devices, looking for the cause of the user's complaints.

The system can adapt to the user by updating the weights of the links. If an option is continually picked after performing one action, the probability of the link is increased, promoting the action. On the other hand, if the user critiques an action, like changing the sound setting when a DVD is inserted, the relative weight of the event will decrease, decreasing the likelihood that the behavior will happen again.

\subsection{Kitchen scheduler}

One of the most stressful and error prone moments in holding dinner parties is assuring that all dishes are prepared on time. A dinner party may involve preparing more than one dish, with limited resources, in a timely manner. To help chefs to plan complex meals, we are building a scheduler for a context aware kitchen [11], using EventNet.

The scheduler first asks EventNet for actions that might not be explicitly expressed in the recipe, and allocates time for their execution. For example, it will recommend taking the meat out of the freezer two hours before starting to cook it, or soaking the vegetables for fifty minutes to defrost them.

Second, the system use ConceptNet [4] 'UsedFor' link to determine what kitchen utensils can be used to perform steps stated in the recipe; "heat water" can be performed by the microwave or the stove. Third, with this information, and the help of a planner, a schedule is shown to the user. In addition, the recipes are loaded on the device, the microwave displays a function names "bake cake" with the cooking time stated in the recipe.

Finally, while cooking, the kitchen watches the chef and uses EventNet plan recognizer to infer the current step of the recipe, and adjusts the plan to the current step. This makes 
the schedule flexible enough so it will be useful even if the chef does not follow the scheduler's advice.

\subsection{Calendar and To-Do list integration}

When planning an event or meeting, there is often a series of prerequisites that are needed. When the user adds a new event in her calendar, a calendar system implemented by Alex Faaborg and Christopher Tsai, using EventNet, asks for the temporal implications. For example: the user wants to schedule a day at the beach, so she sets a new calendar item with the text "go to the beach." The answer that EventNet returns contains "I run at the beach" and "I bring towel." Then, the system looks for specific verbs to identify which portion of the answer is a suitable for a To-Do list and suggests them to the user.

\section{Evaluation}

To evaluate the inference quality, the top ten answers to five random nodes were sent to a group of human judges. In addition, a person unrelated to the project was asked to write ten temporally related events to the same five nodes and the answer was sent to a different group of judges. The difference between the two groups is shown in the next table.

Table 1. EventNet evaluation

\begin{tabular}{|l|l|l|}
\hline & Mean & $\begin{array}{l}\text { Standard } \\
\text { deviation }\end{array}$ \\
\hline EventNet inference & $62 \%$ & 21 \\
\hline Human & $69 \%$ & 19 \\
\hline
\end{tabular}

We found that participants did not always agree on what should be considered temporally related. One of the nodes evaluated was "I study": the answer given by one participant contained the node action "I sleep"; some of the judges had, however, a different line of thought. They thought that, for investing time studding it is necessary give up some sleep.

We also evaluated the coverage of the knowledge by asking five people to write down ten events related to the nodes. We compared their answers to the first hundred EventNet answers. The inference algorithm matched on an average 3.68 out of 10 answers.

The tools described in this paper was conceived to enable interactive applications to integrate better to everyday life. The authors think that the use of EventNet to create successful applications is the best way to test the algorithm. 


\section{Related Work}

We are most interested in Commonsense temporal reasoning, as opposed to formal temporal reasoning. There has been considerable work in Artificial Intelligence in applying formal methods such as first-order logic to temporal reasoning (see, for example [12]), by explicitly introducing variables representing time, time intervals, causality, probability of occurrence, etc.

One example of more formal work in Commonsense theory of time is Allen [13]. His main thesis defines a basic temporal operation - meet(:) - which says that one period of time meets another period without time between them and without sharing - and use it to infer other temporal relations. This allows him to deduce many properties of temporal events which are not guaranteed by the less stringent representations we use. This work differs from the one proposed here since our approach is more concerned with making predictions about likely future or past events, rather than conceptualizing all possible temporal relations. Similarly, work based on networks of known causal relations or quantified known probabilities of events following other events can make much stronger statements than we do about inferred temporal relations. This is a limitation of our approach. As the other, more formal approaches gain traction, we can certainly incorporate some of their techniques to refine our approach.

It is not our goal here to perform this kind of formal reasoning. We are simply interested in representing what an average person might be able to quickly infer (however inaccurately or incompletely) about temporal relations between everyday events, regardless of whether they arise because of causal relations, prerequisites, consequences, shared influences, or contingent probability of occurrence. In the applications we are considering, it is not important to accurately predict the causality or quantify the probabilities of occurrence of the event, though that would certainly be helpful. We believe we have shown that merely by generating a set of plausible possibilities for event occurrence, and using Commonsense reasoning, application context and user interaction to choose from those possibilities, we can significantly improve the behavior of many interactive applications.

\section{Future work}

Other methods of temporal representation are being explored to provide better structure of temporal events. We plan to diversify the semantic sources to improve the expansion. Particularly we are going to use VerbOcean [14] to have a richer semantics to control the creating of the links.

EventNet is part of a more comprehensive effort of create an inference architecture composed by multiple layers of inference each one capable to infer, reflect and correct the knowledge in the layer below [15]. 


\section{References}

1. Singh, Push Lin, T., Mueller, E, Lim, G, Perkins, T., and Zhu, W.: Open Mind Common Sense: Knowledge acquisition from the general public. First International Conference on Ontologies, Databases, and Applications of Semantics for Large Scale Information Systems. Irvine, CA. (2002)

2. McCarthy, John: Programs with Common Sense. In Mechanization of Thought Process, Proceedings of the Symposium of National Physics Laboratory, London, HMOS (1959) 77 84

3. Lenat, Douglas: CYC: A Large-Scale Investment in Knowledge Infrastructure. Communications of the AMC, 38, No. 11, (1995) 33-38

4. Liu, Hugo, Singh, Push: ConceptNet - a practical commonsense reasoning toolkit. BT Technology Journal, (2004) 22(4): 211-226

5. Lieberman, Henry, Liu, Hugo, Singh, Push, and Barry, Barbara. Beating Common Sense into Interactive Applications. AI Magazine 25(4): Winter 2005, 63-76.

6. Pearl, Judea.: Probabilistic Reasoning in Intelligent Systems: Networks of Plausible Inference. San Mateo, CA: Morgan Kaufman. (1988)

7. Singh, Push, Williams, William.: LifeNet: A Propositional Model of Ordinary Human Activity. Proc. Workshop on Distributed and Collaborative Knowledge Capture. Sanibel Island, FL. (2003)

8. Maes Pattie.: How to Do the Right Thing. Connection Science Journal, Vol. 1, No. 3., also MIT AI-Memo \#1180. (1989)

9. Fellbaum, Christiane (Ed.): WordNet: An electronic lexical database, 1998. MIT Press.

10. Cohen, William.: WHIRL: A word-based information representation language. Journal of Artificial Intelligence, (2000) 163-196

11. Bonanni, Leonardo, Lee, Chia-Husun, Selker, Ted.: CounterOntelligence: Augmented Reality Kitchen, 2005. Proc. Computer-Human Interaction, ACM. April 2-7, Portland, Oregon, USA. 2005

12. Lluis Vila: A survey on temporal reasoning in artificial intelligence. AI Communications, 7(1), (March 1994)

13. Allen J., Hayes, P.: A common-sense theory of time. In Proc. Of the $9^{\text {th }}$ International Joint Conference on Artificial Intelligence, Morgan Kaufmannm (1985) 528-531.

14. Chklovski, Timothy and Pantel, Patrick, VerbOcean: Mining the Web for Fine-Grained Semantic Verb Relations, 2004. In Proceedings of Conference on Empirical Methods in Natural Language Processing (EMNLP-04). Barcelona, Spain.

15. Singh, Push, Minsky, Minsky, Eslick, Ian: An architecture for computing common sense. BT Technology Journal. (2004) 22(4): 201-210. 